\title{
Primer reporte de parásitos gastrointestinales en Jirafas (Giraffa camelopardalis reticulata) de cautiverio, Guanacaste, Costa Rica
}

\section{First report of gastrointestinal parasites in Giraffes (Giraffa camelopardalis reticulata) in cap- tivity from Guanacaste, Costa Rica}

\author{
Esteban Palacios Quirós ${ }^{1}$, Ana Eugenia Jiménez Rocha ${ }^{1}$, Tania Pivovarova ${ }^{2}$
}

1 Laboratorio de Parasitología, Escuela de Medicina Veterinaria, Universidad Nacional. Email: teban.vet@hotmail.com, ana.jimenez.rocha@una.cr

2 Regente veterinaria, Ponderosa Adventure Park. Email: taniapivo5@yahoo.com

Recibido: 9 de noviembre de 2017 Corregido: 27 de febrero de 2018 Aceptado: 15 de marzo de 2018

\begin{abstract}
Resumen: La jirafa (Giraffa camelopardalis reticulata), es una especie de ungulado poligástrico, originario del continente africano cuyas poblaciones en cautiverio han aumentado de manera exponencial. Las enfermedades parasitarias se catalogan como una de las principales causas de mortalidad en esta especie. El objetivo del presente trabajo fue determinar las especies y géneros de parásitos gastrointestinales (PGI) en jirafas de cautiverio en Guanacaste, Costa Rica. Se analizó en total 37 muestras de heces de 10 jirafas procedentes de un centro turístico, las cuales se recolectaron de enero a mayo del 2017 en cuatro muestreos, excepto a una de ellas, nacida durante el estudio. El muestreo se realizó al momento de la defecación. Las heces fueron transportadas en frío a una temperatura de $\approx 4{ }^{\circ} \mathrm{C}$, y remitidas al Laboratorio de Parasitología de la Escuela de Medicina Veterinaria de la Universidad Nacional. Las muestras fueron procesadas por técnicas coprológicas (Sheather y sedimentación) para determinar la presencia de huevos de nematodos, trematodos y cestodos; inmunológicas (FasTest ${ }^{\oplus}$ Crypto-Giardia Strip), para detectar parásitos zoonóticos como Cryptosporidium parvum y Giardia duodenalis; McMaster para determinar la carga parasitaria (huevos por gramos de heces= HPG) del grupo Strongylida y el coprocultivo para determinar el porcentaje de larvas infectantes (L3). El 100 $\%$ de las jirafas analizadas fueron positivas a PGI, teniendo toda la población parásitos del grupo Strongylida. Las L3 identificadas correspondieron a 100 \% Haemonchus spp., 10 \% Cooperia spp. y 20 \% Strongyloides papillosus. No se encontraron PGI zoonóticos. El rango de HPG fluctuó de $<50$ a 625 . El porcentaje de larvas infectantes del grupo Strongylida identificadas por coprocultivo fue $100 \%$ (10/10) de Haemonchus spp. y 10 \% (1/10) de Cooperia spp. Únicamente una jirafa tuvo infección mixta por Haemonchus y Cooperia. Este estudio representa el primer reporte de la presencia de PGI en jirafas de Costa Rica, y es el primero que identifica Cooperia spp. y Strongyloides papillosus en el nivel mundial.
\end{abstract}

Palabras claves: jirafas, parásitos gastrointestinales, Costa Rica

Abstract: The giraffe (Giraffa camelopardalis reticulata), is a species of polygastric ungulate, originating from the African continent whose populations in captivity have increased at exponential rate. Parasitic diseases are classified as one of the main causes of mortality in this species. The objective of this work was to determine the species and / or genera of gastrointestinal parasites (GIP) in captive giraffes in Guanacaste, Costa Rica. A total of 37 fecal samples from 10 giraffes from a tourist center were analyzed. The samples were collected from January to May 2017 in four samples, except one of them which was born during the study. Sampling was done at the time of defecation. The feces were transported at a temperature of $\approx 4^{\circ} \mathrm{C}$, and sent to the 


\begin{abstract}
Parasitology Laboratory at the School of Veterinary Medicine of the National University. The samples were processed by coprological techniques (Sheather and sedimentation) to determine the presence of nematode, trematode and cestode eggs; immunological (FasTest ${ }^{\oplus}$ Crypto-Giardia Strip), to detect zoonotic parasites such as Cryptosporidium parvum and Giardia duodenalis; McMaster to determine the parasitic load (eggs per gram of feces $=$ FEC) of the Strongylida group and the coproculture to determine the percentage of infective larvae (L3). $100 \%$ of the giraffes analyzed were positive for GIP, the whole population has parasites of the Strongylida group. The L3 identified corresponded to $100 \%$ Haemonchus spp., $10 \%$ Cooperia spp. and $20 \%$ Strongyloides papillosus. No zoonotic GIP were found. The range of FEC was from $<50$ to 625 . The percentage of infective larvae of the Strongylida group identified by stool culture was $100 \%(10 / 10)$ of Haemonchus spp. and $10 \%(1 / 10)$ of Cooperia spp. Only one giraffe had mixed infection by Haemonchus and Cooperia. This study represents the first report of the presence of GIP in Costa Rican giraffes, and is the first to identify Cooperia spp. and Strongyloides papillosus worldwide.
\end{abstract}

Key words: giraffe, gastrointestinal parasites, Costa Rica

\title{
Introducción
}

Lasjirafas (Giraffa camelopardalis reticulata) son una especie de mamíferos poligástricos y ramoneadores, originarios del continente africano. Las poblaciones de esta especie en cautiverio, han aumentado de manera considerable con el pasar de los años, adquiriendo popularidad en zoológicos y centros de fauna silvestre en África, Asia, Europa y América (Delgado et al. 2003). En jirafas mantenidas en cautiverio, una de las principales causas de mortalidad es el manejo inadecuado de las enfermedades parasitarias (Gómez et al. 1996).

En el nivel mundial los reportes sobre parásitos gastrointestinales (PGI) en jirafas (Girraffa) datan de zoológicos en España: Strongylida (Delgado et al. 2003), Estados Unidos de Norteamérica: Sarcocystis spp. (Kodádková et al. 2010), Japón: Camelostrongylus mentualus, (Matsubayashi et al. 2005), China: Mondetella spp. (Lim et al. 2008). Actualmente existe un único reporte en especímenes de vida libre en la sabana de Kenia: Prabronema skrjabini, Skrjabinema spp., Haemonchus mitchelli y Echinococcus spp. (Chakraborty 1994; Delgado et al. 2003; Matsubayashi et al. 2005; Kodádková et al. 2010). Además, se han reportado en jirafas los parásitos zoonóticos Cryptosporidium spp. y Giardia duodenalis (Delgado et al. 2003). La mayoría de los reportes de PGI en jirafas proceden de hallazgos de necropsia y existe un único estudio donde se ha realizado un análisis coproparasitológico (Lim et al. 2008).

La introducción de especies exóticas en un territorio, exige al médico veterinario establecer protocolos clínicos que prevengan el desarrollo de enfermedades que puedan ocasionar mortalidad en estos grupos de jirafas (Bertelsen et al. 2009). En Costa Rica a partir del año 2007, en el Ponderosa Adventure Park, fue introducida una jirafa hembra preñada, procedente del Wild Animal Life Safari en Ohio, Estados Unidos de Norteamérica.

El desconocimiento en materia médica que se tiene respecto a los grandes mamíferos exóticos en Costa Rica, representa un reto para la salud veterinaria, pues, no se cuenta con una casuística lo suficientemente alta para registrar cuáles parásitos endémicos podrían afectar a estos animales exóticos, y el efecto que los animales domésticos podrían tener sobre estos. 
Los registros médicos para esta especie en nuestro país, así como los programas de desparasitación no son claros, específicos, ni constantes, por lo que es seguro afirmar que no contamos con estatutos de medicina preventiva para las jirafas.

El objetivo de este trabajo fue reportar los parásitos gastrointestinales (PGI) de la única población de jirafas en cautiverio en Costa Rica y discutir los hallazgos encontrados.

\section{Materiales y métodos}

Se analizaron en total 37 muestras de heces de 10 jirafas reticuladas (Giraffa camelopardalis reticulata) ubicadas en el Ponderosa Adventure Park en la provincia de Guanacaste, Costa Rica. Del total de animales muestreados, 4 jirafas se encontraron en aislamiento por maternidad en establos de 250x350 metros cuadrados, y las restantes 6 en pastizales y en contacto con una población de oryx (Oryx beisa). En el centro, el programa de desparasitación no está basado en los hallazgos de las pruebas coprológicas. Las jirafas se desparasitan cada 3 meses con Fenbendazol vía oral (50mg $/ \mathrm{kg}$ ) adicionado al concentrado, y las hembras gestantes son desparasitadas 15 días antes de la fecha del parto. Una vez nacida la cría, esta es incluida en el programa de desparasitación a partir de los tres meses de edad.

Todas las jirafas fueron estudiadas por un periodo de 5 meses (de enero a mayo del 2017), abarcando un total de cuatro muestreos. Un espécimen nacido en el mes de abril, fue muestreado una única vez en mayo, a la edad de 1 mes. Las muestras fecales se recolectaron al momento de la deposición, y fueron debidamente identificadas de acuerdo al registro reportado por el zoológico. Además, fueron transportadas en una hielera, mantenidas a una temperatura de $\sim 4^{\circ} \mathrm{C}$, y remitidas al Laboratorio de Parasitología de la Escuela de Medicina Veterinaria de la Universidad Nacional.

Las muestras fueron analizadas utilizando cinco técnicas de diagnóstico parasitológico. La técnica de flotación en solución hipersaturada de azúcar (Sheather), para la detección de coccidios y nematodos gastrointestinales del grupo Strongylida (Sloss et al. 1995). La técnica comercial de inmucromatografía, FasTest ${ }^{\oplus}$ Crypto-Giardia Strip, para detectar la presencia de parásitos zoonóticos Cryptosporidium parvum y Giardia duodenalis, siguiendo las instrucciones del proveedor (Megacor, Diagnostik). La técnica de sedimentación (Boray), para detectar huevos de tremátodos (Sloss et al. 1995). La técnica de McMaster permitió determinar la carga parasitaria (huevos por gramos de heces, HPG) del grupo Strongylida (Sloss et al. 1995). Finalmente, se utilizó la técnica de coprocultivo para determinar el género o especie de las larvas infectantes (L3) del grupo Strongylida (Sloss et al. 1995).

\section{Resultados}

Del total de jirafas analizadas, el 100 \% fue positiva a PGI. El rango de HPG para huevos de Strongylida fluctuó entre $<50$ a 625 (Cuadro 1). Todos los animales presentaron parásitos del grupo Strongylida, y en dos se determinó Strongyloides papillosus (Cuadro 1 y 2). 
Cuadro 1. Parásitos gastrointestinales en jirafas (Giraffa camelopardalis reticulata) de Ponderosa Adventure Park, Guanacaste, Costa Rica

\begin{tabular}{|c|c|c|c|c|c|}
\hline Identificación & Edad & Sexo & Parásitos & Coprocultivo & $\begin{array}{l}\text { Promedio de HPG } \\
\text { por animal }\end{array}$ \\
\hline 1 & $20 \mathrm{a}^{*}$ & Hembra & Strongylida & Haemonchus spp. & 625 \\
\hline 2 & $11 \mathrm{a}$ & Macho & $\begin{array}{l}\text { Strongylida } \\
\text { Strongyloides papillosus }\end{array}$ & Haemonchus spp. & 150 \\
\hline 3 & $7 \mathrm{a}$ & Hembra & Strongylida & Haemonchus spp. & 100 \\
\hline 4 & $6 \mathrm{a}$ & Macho & $\begin{array}{l}\text { Strongylida } \\
\text { Strongyloides papillosus }\end{array}$ & $\begin{array}{l}\text { Haemonchus spp. } \\
\text { Cooperia spp. }\end{array}$ & $\begin{array}{l}150 \\
<50\end{array}$ \\
\hline 5 & $3 \mathrm{a}$ & Macho & Strongylida & Haemonchus spp. & 200 \\
\hline 6 & $2 \mathrm{a}$ & Hembra & Strongylida & Haemonchus spp. & $<50$ \\
\hline 7 & $2 \mathrm{a}$ & Macho & Strongylida & Haemonchus spp. & 225 \\
\hline 8 & $9 \mathrm{~m}^{* *}$ & Hembra & Strongylida & Haemonchus spp. & 150 \\
\hline 9 & $6 \mathrm{~m}$ & Hembra & Strongylida & Haemonchus spp. & $<50$ \\
\hline 10 & $2 \mathrm{~m}$ & Hembra & Strongylida & Haemonchus spp. & 300 \\
\hline
\end{tabular}

* años ${ }^{* *}$ meses

*** Valores obtenidos como promedio de todos los HPG determinados para cada uno de los muestreos. Intervalo de confianza: 95 \% (límite inferior: 135.75- límite superior: 241.56).

Fuente: Elaboración propia con los datos de la presente investigación.

Las muestras analizadas por coprocultivo mostraron dos géneros de parásitos pertenecientes al orden Strongylida: 100 \% (10/10) Haemonchus y 10 \% (1/10) Cooperia. Solo una jirafa tuvo infección mixta por Haemonchus y Cooperia.

Cuadro 2. Descripción morfológica de los huevos pertenecientes a PGI del grupo Strongylida y de la especie Strongyloides papillosus, detectados en heces de jirafas reticuladas (Giraffa camelopardalis reticulata) del Ponderosa Adventure Park, Guanacaste, Costa Rica

\begin{tabular}{|c|c|}
\hline Grupo / Especie de PGI & Morfología ${ }^{*}$ \\
\hline Strongylida & $\begin{array}{l}\text { Huevos elipsoidales de tipo estrongilido, morulados, de cascarón liso y } \\
\text { delgado. Las dimensiones del mismo son de } 64-106 \mu \mathrm{m} \text { de largo y de } 30- \\
50 \mu \mathrm{m} \text { de ancho. }\end{array}$ \\
\hline Strongyloides papillosus & $\begin{array}{l}\text { Huevos elipsoidales, larvados, cubierta delgada. Tamaño de } 43-60 \mu \mathrm{m} \text { de } \\
\text { largo por } 20-25 \mu \mathrm{m} \text { de ancho. }\end{array}$ \\
\hline
\end{tabular}

${ }^{\star}$ Fuente: Shorroks 2016. 
Rev. Ciencias Veterinarias, Vol. 36, N 1, [27-34], E-ISSN: 2215-4507, enero-junio, 2018

DOI: http://dx.doi.org/10.15359/rcv.36-1.3

URL: http://www.revistas.una.ac.cr/index.php/veterinaria/index

\section{Discusión y conclusiones}

Este es el primer reporte que determina la presencia de Haemonchus, Cooperia y Strongyloides papillosus en jirafas de Costa Rica, y es el primer reporte que identifica Cooperia spp. y Strongyloides papillosus en jirafas reticuladas en el nivel mundial.

En dicho estudio el grupo de nematodos encontrado en mayor proporción fue Strongylida, el cual ha sido reportado en jirafas de vida libre y de cautiverio, debido a que son parásitos cosmopolitas, adaptados a diversas condiciones geoclimáticas y ampliamente distribuidos en rumiantes domésticos y silvestres (Nosal et al. 2016; Shorroks 2016). Las características morfométricas de las larvas examinadas en el coprocultivo permitieron la diferenciación de las L3 de Strongylida e identificación de las especies Haemonchus spp. y Cooperia spp. (Bertelsen et al. 2009; Ming et al. 2010; Taylor et al. 2015).

Haemonchus spp. ha sido descrito ampliamente en rumiantes domésticos (bovinos, ovinos y caprinos) (Jiménez et al. 2007; Jiménez et al. 2010; Taylor et al. 2015) y exóticos (jirafas y okapis) (Shorroks 2016). Es un parásito hematófago del abomaso, altamente patógeno, y la hembra es muy prolífica capaz de producir entre 10000 hasta 20000 huevos por día (Taylor et al. 2015). A pesar de su alto grado de patogenicidad, no hay estudios que asocien la causa de muerte en jirafas con este parásito (Bertelsen et al. 2009). En este estudio el $100 \%$ de las jirafas estuvieron infectadas con Haemonchus, cuya especie no fue determinada, dado que se requiere el uso de técnicas moleculares para hacer la identificación a este nivel (Bertelsen et al. 2009). Sin embargo, no existen estudios sobre el grado de patogenicidad entre especies de este género, en jirafas en cautiverio o vida libre. En ungulados domésticos se han establecido como factores de riesgo el sexo y la edad de los animales (Pato et al. 2013), no obstante, en los exóticos no existen estudios al respecto.

Cooperia spp. es un parásito de abomaso e intestino delgado. Es hematófago con mediana patogenicidad y la hembra es poco prolífica (100 a 200 huevos por día) (Taylor et al. 2015). A pesar de que el $10 \%$ (número de animales) de las jirafas analizadas presentó este parásito, hasta la fecha ningún estudio lo ha reportado en jirafas de vida libre ni de cautiverio. Aunque este parásito es común en rumiantes domésticos, el impacto de las infecciones por agentes de este género es desconocido en poblaciones de jirafas, y no se han realizado estudios que determinen las especies que circulan en los zoológicos de Costa Rica (Fukomoto et al. 1996; Elsheika \& Khan 2011).

Strongyloides papillosus es una especie que habita el intestino delgado y causa parasitosis severas en rumiantes jóvenes durante las primeras semanas de vida (1-6 semanas), la hembra es capaz de producir una cantidad $\sim 3000$ huevos por día, y tiene dos tipos de ciclos de vida uno homogónico y otro heterogónico, los cuales van en función de la edad del animal y de las condiciones ambientales (Taylor et al. 2015). Estas características junto con las vías de transmisión oral, cutánea y galactógena, contribuyen a aumentar la probabilidad de encontrar esta especie, en gran cantidad, en el medio ambiente; sin embargo, solo el $20 \%$ de las jirafas presentó huevos de S. papillosus. Esta especie nunca ha sido descrita en jirafas (Bozkurt et al. 2009). Sin embargo, se ha reportada en otras especies de ungulados silvestres (Mukul-Yerves et al. 2014). Es importante anotar que S. papillosus se ha asociado con problemas respiratorios transitorios por la migración a pulmón en rumiantes domésticos menores a 3 semanas de vida. Los individuos positivos a este nematodo, fueron dos machos adultos de 6 y 11 años de edad, que se mantuvieron en un sistema pastoril con dos individuos jóvenes de 9 meses o de 
menos edad; sin embargo, ninguna de las crías presentó sintomatología clínica parasitaria asociada a esta enfermedad. El hecho de no haber encontrado L3 de S. papillosus en el coprocultivo puede reflejar una baja carga parasitaria (HPG < 50) (Goossens et al. 2005).

En las muestras analizadas, el $80 \%$ de las jirafas presentó valores de HPG > a 50 (100 - 625) y $20 \%$ $\mathrm{HPG}<50$. Este es el primer estudio que determina la carga parasitaria en jirafas y al igual que ocurre en rumiantes domésticos, no existe un umbral de HPG definido como punto para implementar una desparasitación profiláctica. Este valor es útil, si se correlaciona el estado clínico del animal, edad y manejo, siendo estos criterios de gran utilidad para instaurar un adecuado programa de desparasitación (Walker \& Becklund 1971; Goossens et al. 2005). Es importante destacar que los animales con cargas mayores, fueron aquellos mantenidos en aislamiento (cuarentena posnatal), mientras que los que presentaron valores de HPG mayores, se encontraban en pastoreo con cebras (Equus quagga), ñúes (Connochaetes gnou) y una población de venados silvestres (Odocoileus virginianus). Las jirafas comparten con los núes y los venados la misma fauna parasitaria, por ser rumiantes, y a la vez esto podría contribuir a aumentar el HPG de estas, por estar en estrecho contacto con dichas especies.

Los parásitos descritos en este estudio han sido ampliamente analizados en bovinos de Costa Rica (Jiménez et al. 2007; Jiménez et al. 2010). Actualmente no se cuenta con información que correlacione la carga parasitaria de los animales con el estado de salud, pues el desarrollo de cuadros clínicos responde a una dinámica multivariable y dependiente de factores inmunes. En áreas donde los herbívoros silvestres y domésticos comparten tierras de pastoreo, el manejo de parásitos en el ganado puede afectar o ser afectado por la vida silvestre simpátrica debido a la transmisión de especies cruzadas (Walker et al. 2018).

Los resultados obtenidos en este análisis servirán de base para realizar otras investigaciones que permitan, en el futuro, entender los aspectos epidemiológicos de las enfermedades parasitarias que afectan a las especies exóticas en nuestro país.

\section{Agradecimientos}

Al departamento de Gerencia y Regencia Veterinaria del Zoológico Ponderosa Adventure Park, Guanacaste, Costa Rica. Al técnico César Pérez Salas del Laboratorio de Parasitología de la Escuela de Medicina Veterinaria de la Universidad Nacional, por la ayuda brindada durante el procesamiento de las muestras.

\section{Referencias}

Bengis, R.G, Odening, K., Stolte, M., Quandt, S., \& Bockhardt, I. 1998. Three new Sarcocystis species: S. giraffae, S. klaseriensis, S. camelopardalis, from the giraffe in South Africa. Journal of Parasitology 84(3): 562-565. doi: 10.2307/3284724

Bertelsen, M.F., Østergaard, K., Monrad, J., Brøndum, E.T., \& Baandrup, U. 2009. Monodontella giraffae Infection in Wild-caught Southern Giraffes (Giraffa camelopardalis giraffa). Journal of Wildlife Diseases 45(4):1227-1230. doi: doi.org/10.7589/0090-3558-45.4.1227 
Bozkurt, M.F., Alcigir, M.E., Yumusak, N., Kaya, A., Coskan, A.S. \& Kutsal, O. 2009. Hydatid cysts on lungs of two african giraffe. International, Sciences Conferences. Stara Zagora, Bulgaria.

Chakraborty, A. 1994. Occurrence and pathology of Gongylonema infection in captive wild herbivores. Veterinary Parasitology 52(1-2): 163-167. doi: doi.org/10.1016/0304-4017(94)90047-7

Delgado, E., Fonseca, I.P., Fazendeiro, I., Matos, O., Antunes, F., \& Cunha, M. 2003. Cryptosporidium spp. in ruminants at the Lisbon zoo. Journal of Zoo and Wildlife Medicine 34(4): 352-356. doi: doi.org/10.1638/02-049

Elsheika, H., \& Khan, N. 2011. Essentials of Veterinary Parasitology. Ed. University of Nottingham. United Kingdom.

Fukomoto, S., Uchida, T., Ohbayashi, M., Ikebe, Y., \& Sasano, S. 1996. A new host record of Camelostrongylus mentulatus (Nematoda; Trichostrongyloidea) form abomasum of a Giraffe at a Zoo in Japan. Journal of Veterinary Medical Sciences. 58(12):1223-1225. doi: doi.org/10.1292/ jvms.58.12_1223

Gómez, M. S., Vila, T., Feliu, C., Montoliu, I., Gracenea, M., \& Fernández, J. 1996. A Survey for Cryptosporidium spp. in mammals at the Barcelona Zoo. International Journal for Parasitology 26(11): 1331-1333. doi: doi.org/10.1016/S0020-7519(96)00104-X

Goossens, E., Dorny, P., Boomker, J., Vercammen, F., \& Vercruysse, J. 2005. A 12- month survey of the gastrointestinal helmints of antelopes, gazelles and giraffids kept at two zoos in Belgium. Veterinary Parasitology 127(3-4): 303-312. doi: doi.org/10.1016/j.vetpar.2004.10.013

Jiménez, A. E., Montenegro, V. M., Hernández, J., Dolz, G., Maranda, L., Galindo, J., Epe, C., \& Schnieder, T. 2007. Dynamics in infection with gastrointestinal parasites and Dictyocaulus viviparus in dairy and beef cattle from Costa Rica. Veterinary Parasitology 148(3-4): 262-271. doi: doi.org/10.1016/j.vetpar.2007.06.015

Jiménez, A. E., Fernández, A., Alfaro, R., Dolz, G., Vargas, B., Epe, C., \& Schnieder, T. 2010. A crosssectional survey of gastrointestinal parasites with dispersal stages in feces from Costa Rica dairy cattle. Veterinary Parasitology 173 (3-4): 236-246. doi: doi.org/10.1016/j.vetpar.2010.07.013

Kodádková, A., Kváč, M., Ditrich, O., Sak, B., \& Xiao, L. 2010. Cryptosporidium muris in a Reticulated Giraffe (Giraffa camelopardalis reticulata). Journal of Parasitology. American Society of Parasitologists 96(1): 211-212. doi: doi.org/10.1645/GE-2212.1

Krecek, R. C., Boomker, J., Penzhorn, B. L., \& Scheepers, L. 1990. Internal parasites of Giraffes (Giraffa camelopardalis angolensis) from Etosha National Park, Namibia. Journal of Wildlife Diseases 26(3): 395-397. doi: doi.org/10.7589/0090-3558-26.3.395

Lim, Y. A. L., Ngui, R., Shukri, J., Rohela, M., \& Mat Naim, H. R. 2008. Intestinal parasites in various animals at zoo in Malaysia. Veterinary Parasitology 157: 154-159. doi: doi.org/10.1016/j. vetpar.2008.07.015 
Matsubayashi, M., Takami, K., Kimata, I., Nakanishi, T., Hiroyuki, T., Sasai, K., \& Baba, E. 2005. Survey of Cryptosporodium spp. and Giardia spp. infections in various animals at a zoo in Japan. Journal of Zoo and Wildlife Medicine 36(2): 331-335. doi: doi.org/10.1638/04-032.1

Ming, Z., Zhang, L., \& Zhang, L. 2010. Redescription of Monodontella giraffae Yorke et Maplestone, 1926 (Nematoda, Ancylostomatidae) from a giraffe, Giraffa camelopardalis, from Zoo in China, with a discussion on the taxonomic status of Monodontella. Acta Parasitológica 55(1): 66-70. doi: doi.org/10.2478/s11686-010-0012-8

Mukul-Yerves, J. M., Zapata-Escobedo, M. R., Montes-Pérez, R. C., Rodríguez-Vivas, R. I. \& Torres-Acosta, J. F. 2014. Parásitos gastrointestinales y ectoparásitos de ungulados silvestres en condiciones de vida libre y cautiverio el trópico mexicano. Revista Mexicana de Ciencias Pecuarias 5(4): 459-469.

Nosal, P., Kowal, J., Kornas, S., Wyrobisz, A., Skotnicki, J., Basiaga, M. \& Plucińska, N. E. 2016. Endoparasites of exotic ungulates from the Girraffidae and Camelidae families kept ex situ. Annals of Parasitology 62(1): 67-70. doi: doi.org/10.17420/ap6201.34

Pato, F. J., Vázquez, L., Díez-Baños, N., López, C., Sánchez-Andrade, R., Fernández, G., Díez-Baños, P., Panadero, R., Díaz, P. \& Morrondo, P. 2013. Gastrointestinal nematode infections in roe deer (Capreolus capreolus) from the NW of the Iberian Peninsula: Assessment of some risk factors. Veterinary Parasitology 196(1-2): 136-142. doi: doi.org/10.1016/j.vetpar.2013.01.027

Rojas, J. 2008. Resistencia de Haemonchus spp., Trichostrongylus spp., de los bovinos a benzimidazoles (Febendazol, Abendazol), e imidazotiazoles (Levamisol), en los Fundos de la campiña de Cajamarca, Perú. Ed. Universidad de Lima, Perú.

Shorroks, B. 2016. The Giraffe: biology, ecology, evolution and behavior. Ed. Offices. Oxford, United Kingdom.

Sloss, M., Kemp, R. \& Zajac, A. 1995. Veterinary clinical parasitology. $6^{\text {th }}$. Ed. Ames. Iowa. United States of America.

Tadros, G. 1964. On Chabertiella pesteri gen. et sp. nov. (Cyathostomidae) from a Giraffe and on the Validity of Chabertia rishati Akhtar. Journal of Helminthology 1(2): 109-116.

Taylor, M., Coop., R., \& Wall, R. 2015. Veterinary Parasitology. ${ }^{\text {th }}$. Ed. Wiley Blackwell. N. Jersey, U.S.A.

Toro, A., Rubilar, L., Palma, C., \& Pérez, R. 2014. Resistencia antihelmíntica en nematodos gastrointestinales de ovinos tratados con ivermectina y febendazol. Archivos de Medicina Veterinaria 46: 247-252. doi: doi.org/10.4067/S0301-732X2014000200010

Walker, M. L., \& Becklund, W. W. 1971. Occurrence of a cattle eyeworm, Thelazia gulosa (Nematoda: Thelaziidae), in an imported Giraffe in California and T. lacrymalis in a native horse in Maryland. The Journal of Parasitology 57(6): 1362-1363. doi: 10.2307/3278000

Walker, J. G., Evans, K. E., Vineer, H. R., Van Wyk, J. A. \& Morgan, E. R. 2018. Prediction and attenuation of seasonal spillover of parasites between wild and domestic ungulates in an arid mixed-use system. Journal of Applied Ecology: 1-11. doi: doi.org/10.1111/1365-2664.13083 